\title{
Phase field theory of interfaces and crystal nucleation in a eutectic system of fcc structure: I. Transitions in the one-phase liquid region
}

\author{
Gyula I. Tóth \\ Research Institute for Solid State Physics and Optics, P.O. Box 49, H-1525 Budapest, Hungary \\ László Gránásy \\ Brunel Centre for Advanced Solidification Technology, Brunel University, Uxbridge, Middlesex UB8 3PH, \\ United Kingdom and Research Institute for Solid State Physics and Optics, P.O. Box 49, H-1525 \\ Budapest, Hungary
}

(Received 8 September 2006; accepted 1 June 2007; published online 21 August 2007)

\begin{abstract}
The phase field theory (PFT) has been applied to predict equilibrium interfacial properties and nucleation barrier in the binary eutectic system $\mathrm{Ag}-\mathrm{Cu}$ using double well and interpolation functions deduced from a Ginzburg-Landau expansion that considers fcc (face centered cubic) crystal symmetries. The temperature and composition dependent free energies of the liquid and solid phases are taken from CALculation of PHAse Diagrams-type calculations. The model parameters of PFT are fixed so as to recover an interface thickness of $\sim 1 \mathrm{~nm}$ from molecular dynamics simulations and the interfacial free energies from the experimental dihedral angles available for the pure components. A nontrivial temperature and composition dependence for the equilibrium interfacial free energy is observed. Mapping the possible nucleation pathways, we find that the $\mathrm{Ag}$ and $\mathrm{Cu}$ rich critical fluctuations compete against each other in the neighborhood of the eutectic composition. The Tolman length is positive and shows a maximum as a function of undercooling. The PFT predictions for the critical undercooling are found to be consistent with experimental results. These results support the view that heterogeneous nucleation took place in the undercooling experiments available at present. We also present calculations using the classical droplet model [classical nucleation theory (CNT)] and a phenomenological diffuse interface theory (DIT). While the predictions of the CNT with a purely entropic interfacial free energy underestimate the critical undercooling, the DIT results appear to be in a reasonable agreement with the PFT predictions. (C) 2007 American Institute of Physics. [DOI: 10.1063/1.2752505]
\end{abstract}

\section{INTRODUCTION}

The interfacial properties play a central role in the process of crystallization. For example, freezing of an undercooled liquid starts with nucleation, i.e., with the formation of crystal-like heterophase fluctuations, whose size is comparable to the thickness of the interface as observed in atomistic simulations ${ }^{1}$ and predicted by microscopic theory. ${ }^{2}$ According to atomistic simulations for unary and binary systems the solid-liquid interface extends to several molecular layers. ${ }^{1,3}$ Similarly diffuse solid-liquid interfaces have been observed experimentally in liquids and crystallizing glassy systems. ${ }^{4}$ This feature is captured by molecular models based on the density functional approach ${ }^{5}$ and is an inherent property of continuum models based on the square-gradient approximation such as the Cahn-Hilliard type approaches ${ }^{6}$ and various formulations of the phase field theory. ${ }^{7}$ Atomistic simulations ${ }^{1(\mathrm{~d}), 8}$ and continuum theory ${ }^{9}$ imply that competing nucleation pathways may exist in real systems.

Recent work indicates that multiscale approaches based on continuum models with model parameters evaluated from atomistic simulations can quantitatively describe crystal nucleation $^{2(\mathrm{c}), 10}$ and growth. $^{7(\mathrm{~b}), 11}$ For example, the phase field theory has been applied successfully for describing crystal nucleation in the unary Lennard-Jones, ${ }^{10(\mathrm{c})}$ water-ice $^{10(\mathrm{a})-10(\mathrm{c})}$ and hard sphere $\mathrm{e}^{10(\mathrm{~d})}$ systems, and reason- able predictions have been obtained for the close to ideal solution $\mathrm{Cu}-\mathrm{Ni}$ system. ${ }^{10(\mathrm{c})}$ Atomistic simulation performed for the $\mathrm{Cu}-\mathrm{Ni}$ system with suitable model potentials indicate $^{12}$ that the solid-liquid interfacial free energy decreases from the maximum value corresponding to pure $\mathrm{Ni}$ towards the minimum value corresponding to pure $\mathrm{Cu}$ (as we also found ${ }^{13}$ for an ideal solution approximant of the $\mathrm{Cu}-\mathrm{Ni}$ system within the phase field theory). In contrast, little is known about the properties of the crystal-liquid interface in more complex systems, such as the eutectic and peritectic systems that are of outstanding technological importance.

In the present paper, we apply the phase field approach with a recently proposed Ginzburg-Landau free energy to predict the solid-liquid interfacial free energy and the nucleation barrier as functions of the temperature and composition for a eutectic system whose thermodynamic properties are taken from CALPHAD-type calculations. The paper is structured as follows. In Sec. II, we describe the phase field model used in studying the interfacial properties and nucleation together with other cluster models. The materials properties used are compiled in Sec. III. In Sec. IV properties of the equilibrium planar interface and crystal nuclei are investigated as a function of temperature/composition and are compared with experiment and predictions by other theories. A summary of the results is presented in Sec. V. 


\section{APPLIED MODELS}

\section{A. Phase field theory (PFT)}

The local state of the matter is characterized by two fields: The nonconserved phase field $\phi$ that monitors the transition between the liquid $(\phi=1)$ and crystalline phases $(\phi=0)$, and a conserved field, ${ }^{14}$ the coarse-grained mole fraction $c$.

The solid-liquid structural order parameter, associated with the phase field as $m=1-\phi$, might be viewed as the Fourier amplitude of the dominant density wave of the time averaged singlet density in the solid. As pointed out by Shen and Oxtoby, ${ }^{2(\mathrm{~b})}$ if the density peaks in the solid can be well approximated by Gaussians placed at the atomic sites, all Fourier amplitudes can be expressed uniquely in terms of the amplitude of the dominant wave, thus a single structural order parameter suffices in expanding the free energy. In the present study, we neglect the density difference between the solid and liquid phases, which-together with mass conservation-implies that the integral of the composition field over the volume of the system is a constant.

Our starting point is an extended version of the standard binary phase field theory by Warren and Boettinger. ${ }^{15}$ The free energy of the inhomogeneous system is assumed to be a local functional of the phase and composition fields,

$$
F=\int d^{3} r\left\{\frac{\varepsilon_{\phi}^{2} T}{2}(\nabla \phi)^{2}+\frac{\varepsilon_{c}^{2}}{2}(\nabla c)^{2}+f(\phi, c)\right\} \text {. }
$$

Here $\varepsilon_{\phi}$ and $\varepsilon_{c}$ are coefficients to be defined below, $T$ is the temperature, while $f(\phi, c)$ is the local free energy density. The gradient term for the phase field leads to a diffuse crystal-liquid interface, a feature observed both in experiment $^{3}$ and computer simulations. ${ }^{4}$ The local free energy density is assumed to have the form $f(\phi, c)$ $=w(c) T g(\phi)+[1-p(\phi)] f_{S}(c, T)+p(\phi) f_{L}(c, T)$, where different "double well" functions $g(\phi)$ and "interpolation" functions $p(\phi)$ will be used as specified below. The free energy scale $w(c)=\left[(1-c) w_{A}+c w_{B}\right]$ determines the height of the free energy barrier between the bulk solid and liquid states, in terms of the respective values for the pure components, $w_{A}$ and $w_{B}$. The functional forms of $f_{S}(c, T)$ and $f_{L}(c, T)$ are taken from a CALPHAD-type assessment of the system.

Once the functional forms of $g(\phi)$ and $p(\phi)$ are defined, the model parameters $\varepsilon_{\phi}, w_{A}$, and $w_{B}$ can be related to the interfacial free energy $\left(\gamma_{A}\right.$ and $\left.\gamma_{B}\right)$ and interface thickness $\left(\delta_{A}\right.$ and $\delta_{B}$ ) of the equilibrium planar interfaces for pure components $A$ and $B$.

The magnitude of $\varepsilon_{c}$ is less obvious. In the liquid state it can be related to the interaction parameter $\Omega^{L}$ of the liquid as $\varepsilon_{c, L}^{2}=\Lambda^{2}\left(\Omega^{L} / \nu\right)$, where-assuming nearest neighbor interaction - the interaction distance $\Lambda$ is related to the intermolecular distance as $\Lambda=\left(\nu / N_{0}\right)^{1 / 3} / 3^{1 / 2} \cdot{ }^{16}$ Here, $\nu$ is the molar volume and $N_{0}$ is the Avogadro number. In the solid, besides such a chemical contribution, the free energy of phase boundaries contains a physical contribution that includes elastic contributions and depends both on the misorientation of the crystal grains and on the misfit of the crystal structures of the two solid phases. For the sake of simplicity, we consider here only chemical contributions $\varepsilon_{c}^{2}=[1$ $-p(\phi)] \varepsilon_{c, S}^{2}+p(\phi) \varepsilon_{c, L}^{2}=\Lambda^{2}\left\{[1-p(\phi)] \Omega^{L}+p(\phi) \Omega^{S}\right\} / \nu$, where $\Omega^{L}$ and $\Omega^{S}$ have been identified as the composition dependent CALPHAD parameters used in calculating the enthalpy of mixing in the solid and the liquid.

Once the free energy functional is specified, the properties of the equilibrium interface and the critical fluctuations can be found from extremum principles.

\section{Specific double well and interpolation functions}

Here we use two sets of these functions. One of them has been proposed intuitively in an early formulation of the PFT and is in use widely.

a. The "standard" set (PFT/S) These functions are assumed to have the form $g(\phi)=1 / 4 \phi^{2}(1-\phi)^{2}$ and $p(\phi)$ $=\phi^{3}\left(10-15 \phi+6 \phi^{2}\right)$ that emerge from an intuitive formulation of the PFT. ${ }^{17}$

The respective expressions for the model parameters are as follows: $\varepsilon_{\phi}^{2}=6\left(2^{1 / 2}\right) \gamma_{A} \delta_{A} / T_{A}=6\left(2^{1 / 2}\right) \gamma_{B} \delta_{B} / T_{B}, \quad w_{A}$ $=6\left(2^{1 / 2}\right) \gamma_{A} /\left(\delta_{A} T_{A}\right)$ and $w_{B}=6\left(2^{1 / 2}\right) \gamma_{B} /\left(\delta_{B} T_{B}\right)$. Since the interface thickness is about $1 \mathrm{~nm}$ for metals, the assumed independence of $\varepsilon_{\phi}$ of composition leads to the implicit relationship $\gamma_{A} / \gamma_{B}=T_{A} / T_{B}$, which is satisfied with a reasonable accuracy by experimental data, ${ }^{18}$ due to the fact that the solid-liquid interfacial free energy is dominantly of entropic origin (at least for simple liquids). ${ }^{19}$ This model will be denoted as PFT/S.

\section{b. Ginzburg-Landau form for fcc structure (PFT)}

$G L)$ Recently, we have attempted the derivation of these functions for bcc (base centered cubic) and fcc (face centered cubic) structures ${ }^{2(\mathrm{c})}$ on the basis of a single-order-parameter Ginzburg-Landau (GL) expansion that considers the fcc crystal symmetries. This treatment yields

$$
g(m)=(1 / 6)\left(m^{2}-2 m^{4}+m^{6}\right)
$$

and

$$
p(m)=3 m^{4}-2 m^{6},
$$

where $m=1-\phi$, while the expressions that relate the model parameters to measurable quantities are as follows: $\varepsilon_{\mathrm{GL}}^{2}$ $=(8 / 3) C \varepsilon_{\phi}^{2}, w_{A, \mathrm{GL}}=w_{A}(4 C)^{-1}$ and $w_{B, \mathrm{GL}}=w_{B}(4 C)^{-1}$, where $C=\ln (0.9 / 0.1)[3 \ln (0.9 / 0.1)-\ln (1.9 / 1.1)]^{-1}$. This model is denoted henceforth as PFT/GL.

\section{Equilibrium interfaces}

a. Solid-liquid interfaces At a fixed temperature between the eutectic temperature and the melting points of the pure components, solid and liquid phases of appropriate compositions $\left(c_{S}^{e}\right.$ and $c_{L}^{e}$, respectively) coexist. (Below the melting point of the lower melting point component, two such solid-liquid equilibria exist, left and right of the eutectic composition.) The phase and composition field profiles that are realized under such conditions minimize the free energy of the planar interface. This extremum of the free energy functional is subject to the solute conservation constraint discussed above. To impose this constraint one adds the volume integral over the conserved field times a Lagrange multiplier $\lambda$ to the free energy, $\lambda \int d^{3} r c(\mathbf{r})$. The field distributions that 
extremize the free energy obey the appropriate EulerLagrange (EL) equations

$$
\frac{\delta F}{\delta \phi}=\frac{\partial I}{\partial \phi}-\nabla \frac{\partial I}{\partial \nabla \phi}=0
$$

and

$$
\frac{\delta F}{\delta c}=\frac{\partial I}{\partial c}-\nabla \frac{\partial I}{\partial \nabla c}=0,
$$

where $\delta F / \delta \phi$ and $\delta F / \delta c$ stand for the first functional derivative of the free energy with respect to the fields $\phi$ and $c$, respectively, while $I=1 / 2 \varepsilon_{\phi}^{2} T(\nabla \phi)^{2}+1 / 2 \varepsilon_{c}^{2}(\nabla c)^{2}+f(\phi, c)$ $+\lambda c$ is the total free energy density inclusive the term with Lagrange multiplier.

These EL equations have to be solved under the boundary conditions that bulk solid and liquid phases of the equilibrium compositions exist at $z \rightarrow \pm \infty$, respectively. Under such conditions, the Lagrange multiplier can be identified as $\lambda=-(\partial I / \partial c)_{z \rightarrow \pm \infty}=-\left(\partial f_{s} / \partial c\right)\left(c_{S}^{e}\right)=-\left(\partial f_{L} / \partial c\right)\left(c_{L}^{e}\right)$.

Considering the specific form of the free energy functional, the EL equations can be rewritten as

$$
\begin{aligned}
\varepsilon_{\phi}^{2} T \nabla^{2} \phi= & w(c) T g^{\prime}(\phi)+p^{\prime}(\phi)\left[f_{L}(c, T)-f_{s}(c, T)\right] \\
& +\frac{1}{2} \frac{\partial \varepsilon_{c}^{2}}{\partial \phi}(\nabla c)^{2}
\end{aligned}
$$

and

$$
\begin{aligned}
\varepsilon_{c}^{2} \nabla^{2} c= & -\frac{1}{2} \frac{\partial \varepsilon_{c}^{2}}{\partial c}(\nabla c)^{2}-\frac{\partial \varepsilon_{c}^{2}}{\partial \phi}(\nabla \phi \cdot \nabla c)+w^{\prime}(c) \operatorname{Tg}(\phi) \\
& +[1-p(\phi)] \frac{\partial f_{s}}{\partial c}+p(\phi) \frac{\partial f_{L}}{\partial c}-\frac{\partial f_{L}}{\partial c}\left(c_{L}^{\prime \prime}\right),
\end{aligned}
$$

stands for differentiation with respect to the argument.

In the case of planar interface a single spatial variable applies $(z)$, and the EL equations reduce to coupled ordinary differential equations, with boundary conditions prescribing bulk solid, $\phi=0$ and $c=c_{S}^{e}$, and liquid, $\phi=1$ and $c=c_{L}^{e}$, in the far fields, where $c_{S}^{e}$ and $c_{L}^{e}$ are the solidus and liquidus compositions, respectively. The EL equations have been solved numerically by a fifth-order, variable-step Runge-Kutta method. ${ }^{20}$ The interfacial free energy is then evaluated as

$$
\gamma_{\infty}=\int_{-\infty}^{\infty} d z\left\{\frac{\varepsilon_{\phi}^{2} T}{2}(\nabla \phi)^{2}+\frac{\varepsilon_{c}^{2}}{2}(\nabla c)^{2}+\Delta f(\phi, c)\right\},
$$

where $\Delta f(\phi, c)=f(\phi, c)-\left[\partial f_{L} / \partial c\right]_{c \infty}\left(c-c_{\infty}\right)-f_{L}\left(c_{\infty}\right)$ is the free energy density difference relative to the solid or liquid phases that are in equilibrium.

b. Solid-solid interfaces Below the eutectic temperature the $\mathrm{Ag}$ and $\mathrm{Cu}$ rich solid solutions $(\mathrm{S} 1, \mathrm{~S} 2)$ of fcc structure coexist $(\phi=0)$. Neglecting physical effects such as the elastic contributions from the misorientation of the crystal grains and the misfit of the crystal structures of the two solid phases, the composition field distinguishes them in the present formulation. In this approximation, the free energy of the inhomogeneous solid-solid system reads as

$$
F_{s}=\int d^{3} r\left\{\frac{\varepsilon_{c, s}^{2}}{2}(\nabla c)^{2}+f_{s}(c)+\lambda_{s} c\right\},
$$

while a Cahn-Hilliard-type EL equation applies,

$$
\varepsilon_{c, s}^{2} \nabla^{2} c=-\frac{1}{2} \frac{\partial \varepsilon_{c, s}^{2}}{\partial c}(\nabla c)^{2}+\frac{\partial f_{s}}{\partial c}+\lambda_{s} .
$$

For planar interfaces the latter needs to satisfy that $\mathrm{S} 1$ and $\mathrm{S} 2$ solutions of equilibrium composition exist in the far fields $(z \rightarrow \pm \infty)$. The Lagrange multiplier can be then identified as $\lambda_{S}=-\partial f_{S} / \partial c \quad(z= \pm \infty)$. After trivial algebraic manipulations the boundary thickness and phase boundary energy can be determined as described in Ref. 16.

\section{Barrier for crystal nucleation}

Crystallization of nonequilibrium liquids starts with nucleation, a process in which crystal-like fluctuations appear, whose formation is governed by the free energy gain when transferring molecules from liquid to the crystal and the extra free energy $\gamma$ needed to create the crystal-liquid interface. ${ }^{1,21-23}$ The fluctuations larger than a critical size have a good chance to reach macroscopic dimensions, while the smaller ones dissolve with a high probability. Being in unstable equilibrium, the critical fluctuation (the nucleus) can be found as an extremum (saddle point) of this free energy functional, ${ }^{2,10,21,23}$ subject again to the constraint of mass conservation discussed above. The field distributions that extremize the free energy obey Eq. (3). ${ }^{2,10,21,23}$ However, these EL equations are to be solved now assuming an unperturbed liquid $\left(\phi=1, c=c_{\infty}\right)$ in the far field, while for symmetry reasons zero field gradients appear at the center of the fluctuations. Under such conditions, the Lagrange multiplier can be identified as $\lambda=-(\partial I / \partial c)_{r \rightarrow \infty}=-\left(\partial f_{L} / \partial c\right)\left(c_{\infty}\right)$.

Assuming spherical symmetry-a reasonable approximation considering the weak anisotropy of the crystal-liquid interface of simple liquids - the EL equations [Eq. (3)] have been solved numerically under the following boundary conditions: zero field gradients at $r=0$ and unperturbed liquid in the far field $(r \rightarrow \infty)$. Since $\phi$ and $d \phi / d r$ and $c$ and $d c / d r$ are fixed at different locations, the central values of $\phi$ and $c$ that satisfy $\phi \rightarrow \phi_{\infty}=0$ and $c \rightarrow c_{\infty}$ for $r \rightarrow \infty$ have been determined iteratively. Having determined the solutions $\phi(r)$ and $c(r)$, the nucleation barrier $W^{*}$ has been obtained by inserting these solutions into

$$
W^{*}=\int_{0}^{\infty} 4 \pi r^{2}\left\{\frac{\varepsilon_{\phi}^{2} T}{2}(\nabla \phi)^{2}+\frac{\varepsilon_{c}^{2}}{2}(\nabla c)^{2}+\Delta f(\phi, c)\right\} d r,
$$

where $\Delta f(\phi, c)=f(\phi, c)-\left[\partial f_{L} / \partial c\right]_{c^{\infty}}\left(c-c_{\infty}\right)-f_{L}\left(c_{\infty}\right)$ is the free energy density difference relative to the unperturbed liquid. Provided that the model parameters $\varepsilon_{\phi}, w_{A}$, and $w_{B}$ have been evaluated from the thickness and free energy of the equilibrium planar interface and $\varepsilon_{c}$ from the interaction parameter, the nucleation barrier $W^{*}$ in the undercooled state can be calculated without adjustable parameters.

\section{Tolman length}

The interfacial free energy of small crystalline particles is expected to depend on size, due to the reduction of the 
average number of solid neighbors for molecules on curved surfaces relative to that on a planar interface. The analogous phenomenon in small liquid droplets has been studied extensively. ${ }^{24,25}$ In the widely acknowledged thermodynamic theory of Tolman, ${ }^{26}$ the size dependence of the surface tension is given in terms of the Tolman length, ${ }^{27} \delta_{T}=R_{e}-R_{p}$ (the distance of the equimolar surface $R_{e}$ from the surface of tension $R_{p}$, for definitions see Refs. 28 and 29) as $\gamma$ $\approx \gamma_{\infty} /\left(1+2 \delta_{T} / R_{p}\right)$, where $\gamma_{\infty}$ is the surface tension for planar geometry. Although a rigorous derivation of these notions is unavailable for crystallites, a quantity analogous to $\delta_{T}$ has been evaluated from atomistic simulations. ${ }^{22(\mathrm{~b})}$ It decreases with increasing size of the fluctuations (with a cluster size for crystal-like fluctuations determined using structural criteria to distinguish "liquid-type" and "solid-type" molecules). ${ }^{22(b)}$ It is of considerable interest to see whether the predictions of the present approach are consistent with this result.

Since in the only attempt to evaluate the Tolman length $\delta_{T}$ for the solid-liquid interface from atomistic simulations the cluster size has been determined using structural criteria to distinguish liquid-type and solid-type molecules, ${ }^{22(b)}$ herein we evaluate the equimolar surface analogously from the structural order parameter profile $m(r)$. We adopt the Gibbs surface $R_{m}$ for the order parameter as the equimolar surface [Gibbs surface, the position of the step function, whose amplitude and volumetric integral is the same as the original profile $\left.m(r),(4 \pi / 3) R_{m}^{3} \cdot m(0)=\int_{0}^{\infty} d r \cdot 4 \pi r^{2} \cdot m(r)\right]$. The radius of the surface of tension is in turn evaluated via the expression $R_{p}=\left[3 W^{*} /(2 \pi \Delta g)\right]^{1 / 3}$ deduced for liquid droplets, ${ }^{30}$ where $\Delta g(>0)$ is the volumetric free energy difference between the melt and the crystal. Previous work indicates that for symmetrical free energy wells the Tolman length is zero, ${ }^{25(a)}$ while for the asymmetric case the sign of $\delta_{T}$ depends on whether the solid or the liquid side of the free energy is steeper. ${ }^{10(b)}$

\section{B. Classical nucleation theory (CNT)}

For the sake of comparison, we calculate the free energy of critical fluctuations from the classical droplet model,

$$
W_{\mathrm{CNT}}=(16 \pi / 3) \gamma^{3} / \Delta g^{2},
$$

where $\gamma$ is the solid-liquid interfacial free energy between the nucleus and the undercooled liquid (see, e.g., Ref. 31). Here we adopt the following approximation to calculate the solid-liquid interface free energy as a function of composition and temperature in the undercooled state: We assume here that the solid-liquid interfacial free energy is essentially of entropic origin as trivially happens for the hard sphere system and is observed for other simple liquids as the Lennard-Jones system in atomistic simulations. ${ }^{32}$ Accordingly, it is made to scale with temperature and entropy as follows:

$$
\gamma=\gamma_{\infty}(c)\left(T / T_{\mathrm{eq}}\right),
$$

where $\gamma_{\infty}(c)$ is the equilibrium interfacial free energy for a planar interface at temperature $T_{\text {eq }}$ between a liquid of composition $c\left(T_{\text {eq }}\right)$ and a solid of the corresponding solidus composition. Equation (10) can also be viewed as a generaliza- tion of the negentropic model of Spaepen and Meyer. ${ }^{33}$

\section{Diffuse interface theory (DIT)}

The diffuse interface theory (DIT) relies on the assumptions that bulk properties exist at least at the center of critical fluctuations and that the distance between the surfaces of zero excess enthalpy and zero excess entropy is independent of cluster size. ${ }^{34}$ The height of the nucleation barrier reads as

$$
W_{\text {DIT }}=(4 \pi / 3) \delta^{3} \Delta g \psi,
$$

where $\delta=\gamma_{\infty} \nu / \Delta H_{f}$ is the characteristic interface thickness, $\Delta H_{f}(>0)$ the molar heat of fusion, $\psi=2(1+q) \xi^{-3}-(3$ $+2 q) \xi^{-2}+\xi^{-1}, q=(1-\xi)^{1 / 2}$, and $\xi=\Delta g / \Delta h$, while $\Delta h(>0)$ is the volumetric enthalpy difference between the solid and liquid. Note that the thickness parameter $\delta$ as defined above is only usually a fraction of the interface thickness, and has a different critical exponent than the correlation length. This model has been tested extensively. ${ }^{35,36}$ It leads to an improved agreement with vapor condensation experiments relative to the classical theory ${ }^{35}$ and in the range of interest reproduced $W^{*}$ predicted by density functional theory to a high accuracy. $^{25(\mathrm{~d})}$ The DIT also proved consistent with crystal nucleation experiments on a broad variety of substances including liquid metals, oxide glasses, and hydrocarbons, ${ }^{36}$ and with atomistic simulations. ${ }^{2(c), 37}$ Provided that the interface free energy and the thermodynamic properties $(\Delta g$ and $\Delta h)$ are known, the nucleation barrier can be calculated without adjustable parameters.

\section{Steady state nucleation rate}

Having determined the height of the nucleation barrier, the steady state nucleation rate (the net number of critical fluctuations formed in unit volume and time), $J_{S S}$, can be calculated as

$$
J_{\mathrm{ss}}=J_{0} \exp \left\{-W^{*} / k T\right\}=b D \Lambda^{-2}\left(i^{*}\right)^{2 / 3} N_{1} Z \exp \left\{-W^{*} / k T\right\},
$$

here $J_{0}$ is the nucleation prefactor, $b=24$ a geometrical factor, $i^{*}$ the number of molecules in the critical fluctuation, $D$ the self-diffusion coefficient, and $N_{l}$ the number density of molecules in the liquid, while $Z=\left\{\left|d^{2} W / d i^{2}\right|_{i}{ }^{*} /(2 \pi k T)\right\}^{1 / 2}$ $\approx 0.01$ is the Zeldovich factor that accounts for the dissolution of critical clusters. This form of the nucleation prefactor has been deduced on the basis of the classical kinetic theory $^{31}$ that has been verified via comparison with experiments on transient nucleation in oxide glasses. ${ }^{38}$ Recent molecular dynamics calculations indicate, however, that it might be about two orders of magnitude too low. ${ }^{39}$

\section{PHYSICAL PROPERTIES}

In the present calculations the free energies of the bulk phases have been taken from a CALPHAD-type assessment of the Ag-Cu system used in Ref. 40. The phase diagram we calculated using these data is presented in Fig. 1. A typical free energy surface corresponding to $T=900 \mathrm{~K}$ is displayed in Fig. 2. The interaction parameters used in calculating the coefficient of the square-gradient term for the composition 


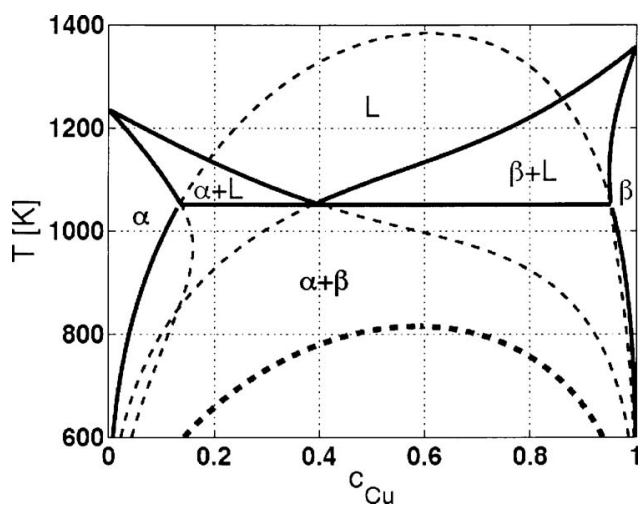

FIG. 1. Phase diagram of the Ag-Cu system calculated on the basis of the free energy functions from CALPHAD-type calculations in Ref. 41. Note the metastable extensions of the liquidus, solidus, and solid-solid coexistence lines (light dashed), and the metastable liquid-liquid coexistence line (heavy dashed) at the lower part of the diagram. The phases appearing in the phase diagram are denoted as follows: $\alpha, \mathrm{Ag}$ rich fcc solid solution; $\beta, \mathrm{Cu}$ rich fcc solid solution; and $L$, liquid.

field have been identified as $\Omega^{L}=A_{L}+A 1_{L}(1-2 c)$ and $\Omega^{s}$ $=A_{F}+A 1_{F}(1-2 c)$, respectively, with the notations of Table I of Ref. 40.

To study the effect of the mixing contributions on the interfaces (except the ideal mixing entropy), the respective coefficients $A_{L}, B_{L}, A 1_{L}, B 1_{L}$ and $A_{F}, B_{F}, A 1_{F}, B 1_{F}$ in Table I of Ref. 40 have been multiplied by the factors $\kappa=0,1 / 3$, $1 / 2$, and $3 / 4$. The corresponding phase diagrams are shown in Figs. 3(a)-3(d), which display a transition from an idealsolution-type phase diagram into a eutectic one.

The free energy of the equilibrium solid-liquid interface of pure Ag is $\gamma=172 \mathrm{~mJ} / \mathrm{m}^{2}$, a value evaluated from dihedral angle measurements. ${ }^{41}$ For $\mathrm{Cu}$ the undercooling experiments imply $\gamma=177,200,195 \mathrm{~mJ} / \mathrm{m}^{2}$ [see Refs. 42(a)-42(c), respectively]. These values are somewhat lower than those from dihedral angle measurements $\left(223\right.$ and $232 \mathrm{~mJ} / \mathrm{m}^{2}$, Refs. 41 and 43, respectively). Herein, we use the average of the results from dihedral angle measurements, $\gamma$ $=227 \mathrm{~mJ} / \mathrm{m}^{2}$.

The 10\%-90\% interface thickness for $\mathrm{Ag}$ has been assumed to be $d_{\mathrm{Ag}}=1 \mathrm{~nm}$ in agreement with the atomistic simulations for metals. ${ }^{3(\mathrm{~d})-3(\mathrm{f}), 44}$ As mentioned, in the present formulation of the binary phase field theory the restriction $\gamma_{A} \delta_{A} / T_{A}=\gamma_{B} \delta_{B} / T_{B}$ applies. ${ }^{15}$ As a result, we are not free to

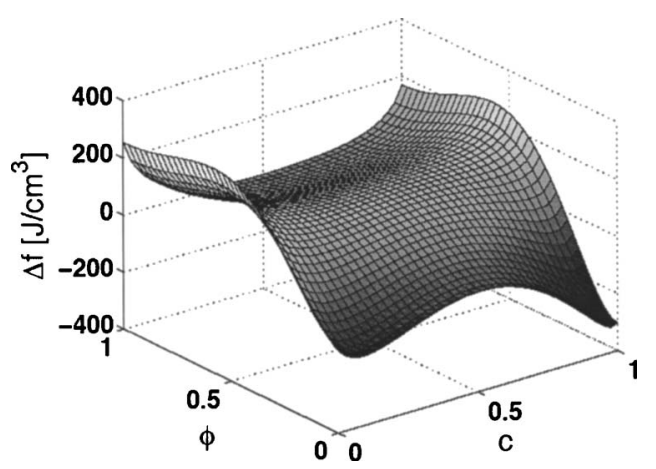

FIG. 2. Free energy density surface at $T=900 \mathrm{~K}$ in the phase field theory, counted relative to a homogeneous liquid of composition of $c=0.5$. Note the different depths of the two solid minima (at $\phi=0$ ).
TABLE I. Physical properties of $\mathrm{Ag}$ and $\mathrm{Cu}$ used in computations. Notation: $\eta=A \exp [B / R T]$, where $R$ is the gas constant.

\begin{tabular}{lcc}
\hline \hline & $\mathrm{Ag}$ & $\mathrm{Cu}$ \\
\hline$T_{m}(\mathrm{~K})$ & 1235 & 1357 \\
$\Delta H_{f}(\mathrm{~kJ} / \mathrm{mol})$ & 11.945 & 13.054 \\
$\gamma\left(\mathrm{mJ} / \mathrm{m}^{2}\right)$ & $172^{\mathrm{a}}$ & 227 \\
$d(\mathrm{~nm})$ & 1.0 & 0.834 \\
$\rho_{s}\left(\mathrm{~g} / \mathrm{cm}^{3}\right)$ & $9.82^{\mathrm{a}}$ & $8.37^{\mathrm{a}}$ \\
& & \\
$A(\mathrm{mP} \mathrm{s})$ & $V^{2}$ & $0.5269^{\mathrm{b}}$ \\
$B\left(\mathrm{~J} / \mathrm{mol}^{2}\right)$ & $0.4301^{\mathrm{b}}$ & $22460^{\mathrm{b}}$ \\
\hline \hline
\end{tabular}

${ }^{\mathrm{a}}$ Reference 32.

${ }^{\mathrm{b}}$ Reference 45 .

choose the interface thickness for $\mathrm{Cu}$. The value that follows from this relationship is $d_{\mathrm{Cu}}=0.834 \mathrm{~nm}$ that is also close to values from atomistic simulations. Considering that the interface thickness is roughly a nanometer for metals, this relationship implies that the interfacial free energy of elements is roughly proportional to their melting point, as indeed argued and seen recently. ${ }^{18,19}$
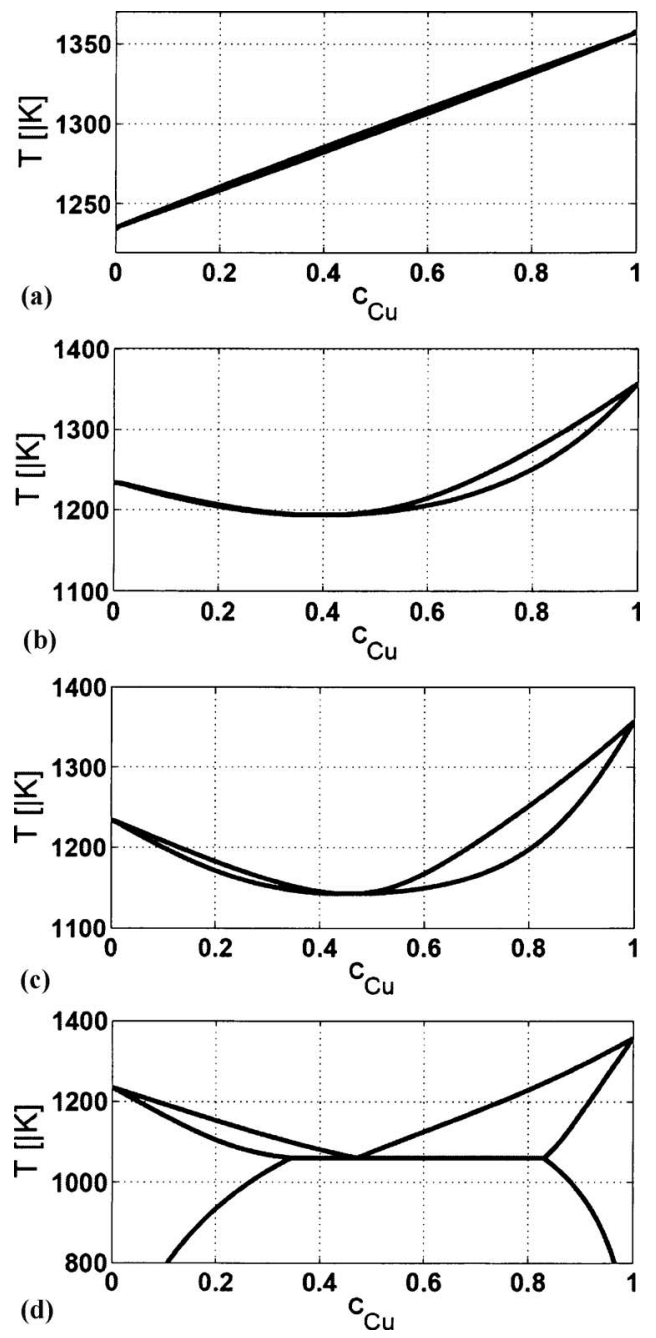

FIG. 3. Model phase diagrams obtained by multiplying the coefficients $A_{L}, B_{L}, A 1_{L}, B 1_{L}$ and $A_{F}, B B_{F}, A 1_{F}, B 1_{F}$ of Ref. 40 by the factors $\kappa=0,1 / 3$, $1 / 2$, and $3 / 4$ (from top to bottom, respectively). 

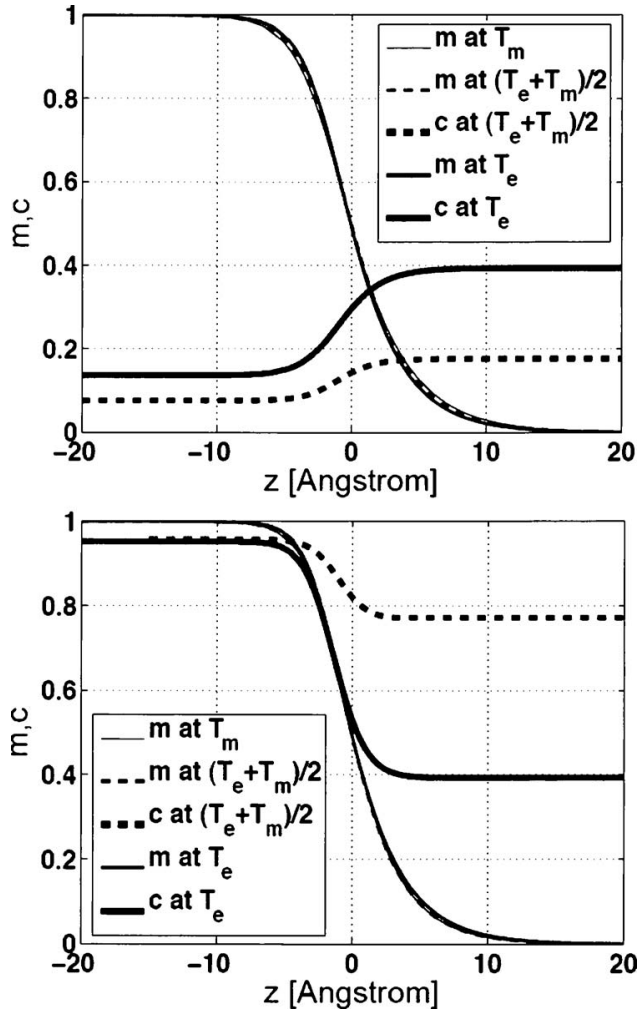

FIG. 4. Cross-interfacial order parameter and composition profiles at the melting point of the pure components $\left(T_{m}\right)$ at the eutectic temperature $\left(T_{e}\right)$ and midway in between $\left[\left(T_{e}+T_{m}\right) / 2\right]$ on the $\mathrm{Ag}$ rich side (upper panel), and on the $\mathrm{Cu}$ rich side (lower panel) for $\kappa=1$.

The calculations have been performed using the average molar volume $\nu=9.29 \mathrm{~cm}^{3}$, calculated form molar weights and the high temperature mass densities of the crystalline phase taken from Ref. 41.

The self-diffusion coefficient of the liquid alloy compositions has been approximated by linearly interpolating between the diffusion coefficients of the pure constituents, which were evaluated in turn from Arrhenius expressions, fitted to the measured viscosities $\eta,{ }^{45}$ using the StokesEinstein relationship, $D=k T /(3 \pi \Lambda \eta)$. This might somewhat underestimate $D .^{45}$

The relevant physical properties are compiled in Table I.

\section{RESULTS AND DISCUSSION}

In this section, first the equilibrium planar solid-liquid interfaces are studied. This is followed by an investigation of the properties of crystal nuclei and the possible nucleation pathways. Since in the GL approach the double-well and interpolation functions are derived on a physical basis, we perform most of these investigations using this version of the PFT (PFT/GL). Nevertheless, for comparison, some nucleation properties are also calculated using other approaches, including the PFT with the standard interpolation and double well functions (PFT/S) and with a binary generalization of the classical droplet model (CNT).

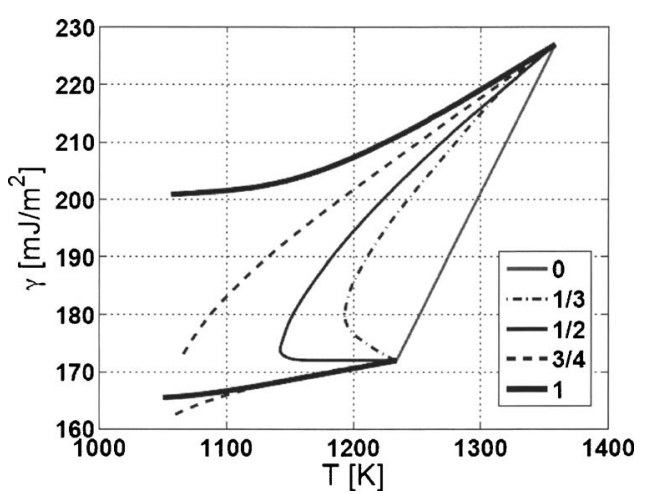

FIG. 5. Temperature dependence of the free energy of the equilibrium planar interfaces between solids and liquids, whose compositions are given by the solidus and liquidus lines shown in Figs. 1 and 2. The numbers in the legend indicate the $\kappa$ factors by which the interaction parameters, $A_{L}, B_{L}, A 1_{L}, B 1_{L}$, and $A_{F}, B_{F}, A 1_{F}, B 1_{F}$ of Ref. 40 , have been multiplied. Note the essentially linear interpolation between the free energies of the pure constituents in the ideal solution case (multiplier $=0$ ).

\section{A. Equilibrium interfaces \\ 1. Solid-liquid interfaces}

Typical cross-interfacial order parameter and composition profiles corresponding to three different temperatures are shown in Fig. 4 for the $\mathrm{Ag}$ and $\mathrm{Cu}$ rich sides of the phase diagram. We find that the 10\%-90\% interface thickness for the order parameter and composition profiles is essentially independent of the temperature on both sides. This prediction is in agreement with the results of atomistic simulations for simple liquids. ${ }^{46}$ The asymmetric order parameter profiles are in a qualitative agreement with results from a detailed density functional theory of fcc solidification. ${ }^{2(b), 47}$

The free energies associated with the equilibrium interfaces calculated isothermally between the solids and liquids of compositions given by the solidus and liquidus curves in Fig. 1 are presented as a function of temperature and liquid composition in Figs. 5 and 6. While in the ideal solution limit $(\kappa=0)$, the interfacial free energy interpolates roughly linearly between the pure components, in the case of larger multipliers, we find a more complex behavior. For $\kappa=0,1 / 4$ and $1 / 3$, a $C$-type curve connects the vales for copper and silver. The $C$-type curve breaks up into two branches for

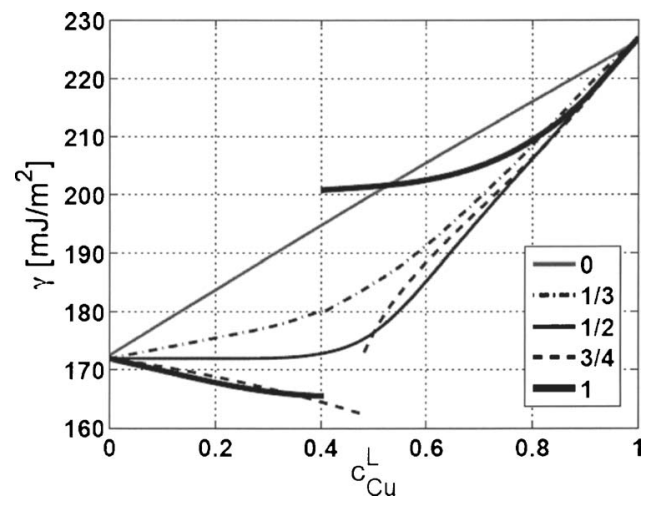

FIG. 6. Free energy of the equilibrium planar solid-liquid interface vs the composition of the liquid phase as a function of the $\kappa$ multiplier by which the interaction parameters $\left(A_{L}, B_{L}, A 1_{L}, B 1_{L}\right.$ and $A_{F}, B_{F}, A 1_{F}, B 1_{F}$ of Ref. 40$)$ have been multiplied. 

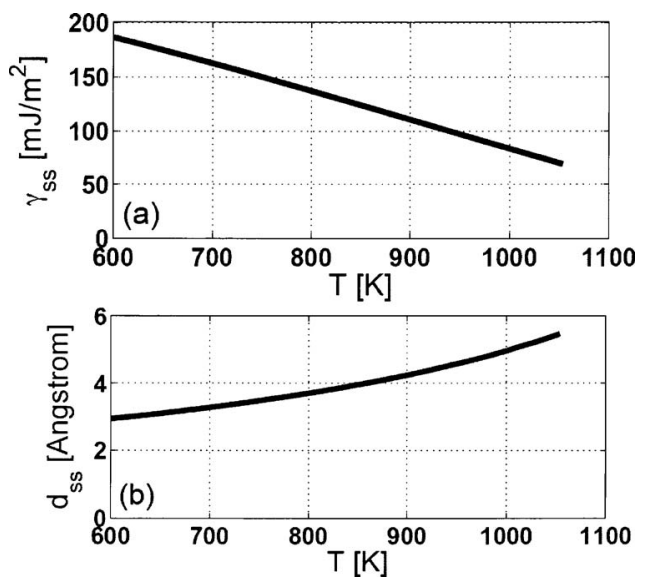

FIG. 7. Properties of the solid-solid phase boundary as a function of temperature: (a) interfacial free energy and (b) 10\%-90\% interface thickness.

larger $\kappa$ values (e.g., for $3 / 4$ and 1 ), due to the appearing eutectic temperature in the respective phase diagrams. In the $\mathrm{Ag}-\mathrm{Cu}$ system, we find that the solid-liquid interface free energy has a positive temperature coefficient on both branches, however, its magnitude is larger for the copper branch. Apparently, the curves level off near the eutectic temperature.

The same data are shown as a function of liquid composition in Fig. 6. A nontrivial behavior is seen that can be associated with the increasingly complex chemical contribution as the mixing free energy contributions increase.

\section{Solid-solid interfaces}

The phase boundary energy $\gamma_{\mathrm{SS}}$ and the phase boundary thickness $d_{\mathrm{PB}}$ for $\kappa=1$ (Fig. 1) are shown as a function of temperature in Fig. 7. As expected on the basis of the CahnHilliard theory, ${ }^{16}$ the phase boundary energy decreases, while the phase boundary thickness increases towards the respective critical point. Note that the full phase boundary thickness (typically $\sim 2 d_{\mathrm{SS}}$ ) extends to roughly $0.6-1 \mathrm{~nm}$ in the temperature range below the eutectic temperature and that $\gamma_{\mathrm{SS}}$ is entirely of chemical origin. This should be corrected for a contribution emerging from the structural/orientational mismatch between the two phases.

\section{B. Crystal nuclei}

\section{Nucleation in PFT with GL free energy}

The radial order parameter and composition profiles are shown in Fig. 8 for the pure constituents and for the central composition $\left(c_{\infty}=0.5\right)$ as a function of undercooling. The height of the nucleation barrier is presented as a function of undercooling in Fig. 8(d). As expected, nucleation is slow in the vicinity of the eutectic composition due to the diminishing undercooling/driving force. We observe that the interface of fcc nuclei sharpens with increasing undercooling (Fig. 9) as also observed in the density functional theory of fcc crystal nucleation. ${ }^{2(b)}$ This change of the interface thickness is probably associated with the restructuring of the interface due to curvature. For small driving forces (large nuclei, $R_{m}$ $\rightarrow \infty$ ) we find $\left[\phi \rightarrow 0\right.$ and $c \rightarrow c_{s}$ (solidus) $]$ at the center of nuclei $(r=0)$. With increasing undercooling, however, one
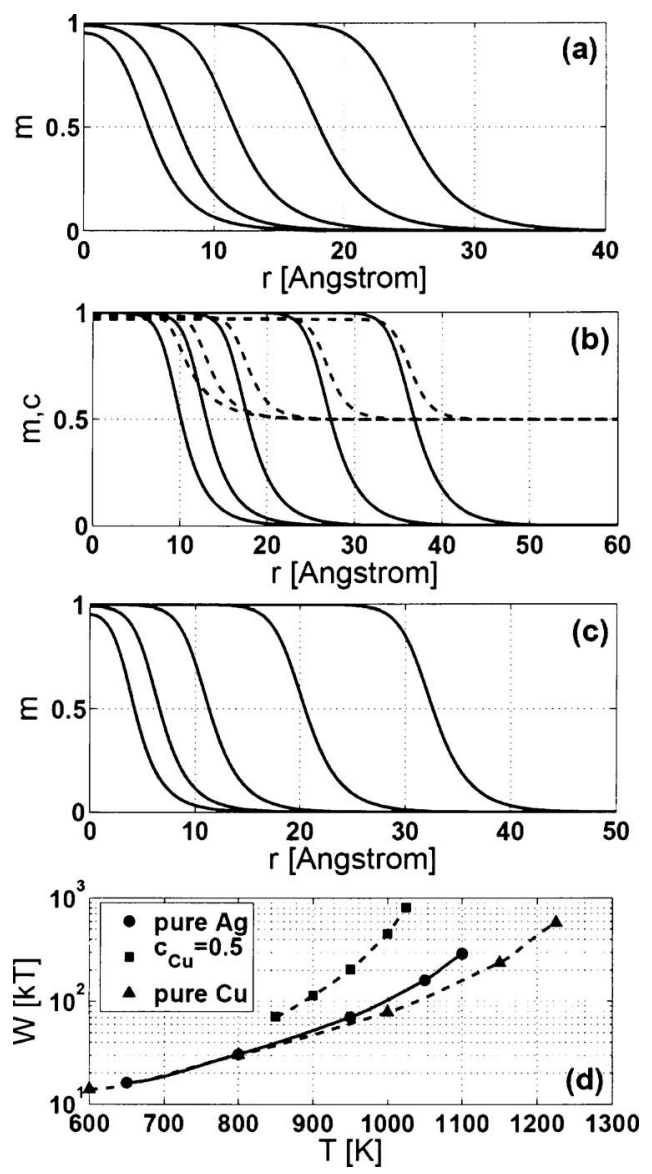

FIG. 8. Properties of the critical fluctuations (nuclei). (a) Radial order parameter profiles for pure $\mathrm{Ag}$ at temperatures (from left to right) $T=650,800$, 950, 1050, and $1100 \mathrm{~K}$; (b) for liquid composition $c=0.5$ at $T=850,900$, 950,1000 , and $1025 \mathrm{~K}$ [the respective composition profiles are also shown (dashed)]; and (c) for pure $\mathrm{Cu}$ at $T=600,800,1000,1150$, and $1225 \mathrm{~K}$. The respective free energies of formation are also shown as a function of temperature [panel (d)].

can reach a regime, where the nucleus is made of all interface (the size of the nucleus is comparable to the interface thickness), i.e., the value of the phase field and composition at the center of the nucleus deviate from the "bulk" values characteristic to large particles [see Figs. 8(a) and 8(c)]. Here we define bulk in the sense that interface thickness is negligible relative to the size of the particle. Assuming a sharp interface, we are able to assign bulk properties for all undercoolings and initial liquid compositions. In this sense, the

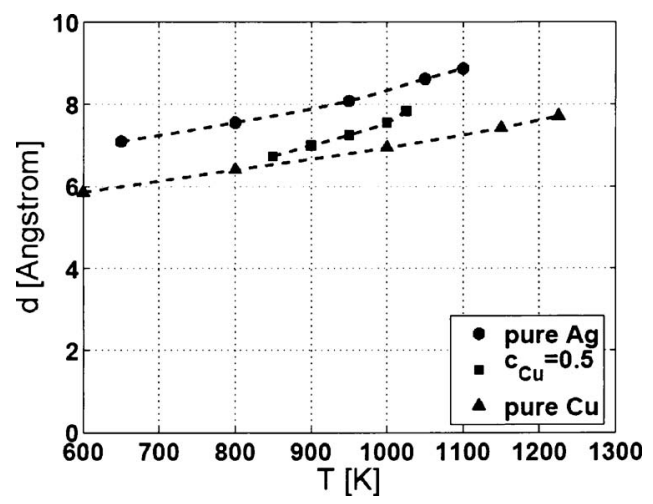

FIG. 9. The 10\%-90\% interface thickness for the nuclei shown in Fig. 8. 


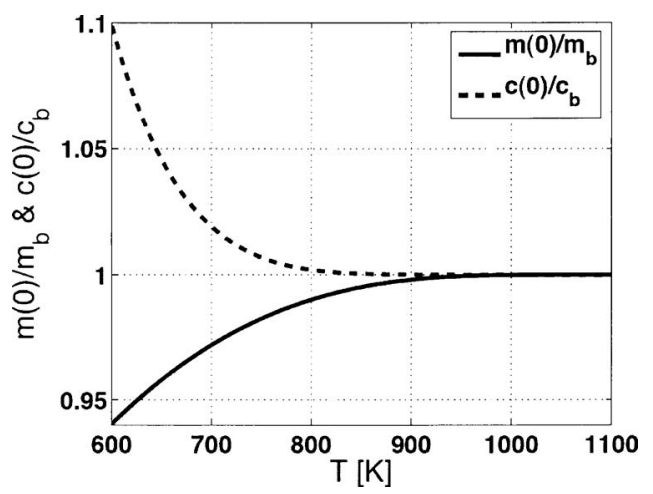

FIG. 10. Normalized structural order parameter $\left[m(r=0) / m_{b}\right]$ and composition $\left[c(r=0) / c_{b}(T)\right]$ values at the center of the nuclei as a function of temperature. Normalization has been done by using the "bulk" values $m_{b}$ $=1$ and $c_{b}(T)$ that maximizes the driving force relative to the initial liquid of composition $c_{\infty}=0.05$.

bulk value of solid composition $c_{b}$ at a given $T$ and $c_{\infty}$ is defined via maximizing the driving force (i.e., $c_{b}$ is the solid composition, for which the tangent of the Gibbs free energy has the same slope as for the initial $\operatorname{liquid}^{48}$ ), while in our formulation, $m_{b}=1$ stands for the bulk crystalline state. A comparison of the concentration and phase field values realized at the center of the fluctuations relative to their bulk counterpart is shown as a function of temperature at $c_{\infty}$ $=0.05$ in Fig. 10. We observe nonbulk physical properties at the center of the critical fluctuations (nuclei) only at extreme supercoolings that are not easily accessible for experiment.

A contour map of nucleation barrier heights is shown in Fig. 11 that also displays the region of nucleation rates available for the usual undercooling techniques, defined here as $10^{-2} \mathrm{~cm}^{-3} \mathrm{~s}^{-1}<J_{\mathrm{ss}}<10^{8} \mathrm{~cm}^{-3} \mathrm{~s}^{-1}$. This region seems to follow closely the iso- $W^{*}$ lines and lies roughly parallel to the liquids line, however, by about $300 \mathrm{~K}$ lower. For comparison, maximum undercoolings achieved by various experimenters are also displayed. ${ }^{42(\mathrm{c}), 49-52}$ These points fall well

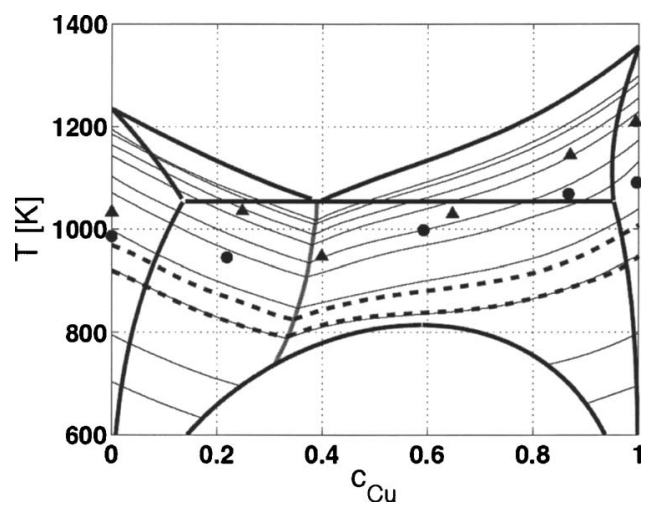

FIG. 11. Contour map of the height of the nucleation barrier as a function of temperature and composition of the initial liquid for the $\mathrm{Ag}-\mathrm{Cu}$ system, as predicted by the PFT with a GL free energy. From bottom to top, the iso- $W^{*}$ lines correspond to $20,30,60,100,200,300,600,1000,2000,3000 k T$, respectively. The nucleation barriers for the $\mathrm{Ag}$ rich and $\mathrm{Cu}$ rich solutions are equal along the gray line starting from the eutectic point. For comparison, maximum undercooling data from experiments are also presented [(full circles) Refs. 42(c) and 49-51 and (full triangle) Ref. 52]. The dashed lines indicate locations where the steady state nucleation rates are $J_{\mathrm{SS}}$ $=10^{-2} \mathrm{~cm}^{-3} \mathrm{~s}^{-1}$ (upper line) and $10^{8} \mathrm{~cm}^{-3} \mathrm{~s}^{-1}$ (lower line).

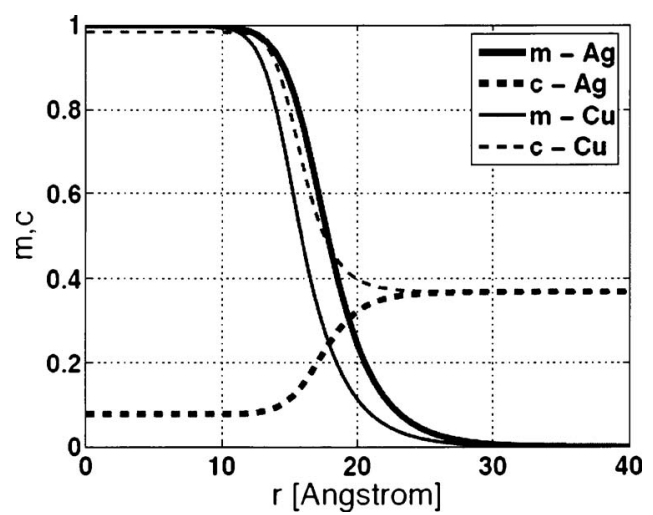

FIG. 12. Radial concentration (dashed) and order parameter (solid) profiles for the $\mathrm{Ag}$ rich nucleus (heavy lines) and the $\mathrm{Cu}$ rich nucleus (light lines) at $T=900 \mathrm{~K}$ and $c=0.3685$, where the nucleation barrier height is equal for the two solutions.

above the region of observable nucleation rates for homogeneous nucleation, implying that the nucleation mechanism in these experiments was most probably heterogeneous nucleation on foreign particles/surfaces.

We observe essentially two types of nuclei in the system, a silver rich and a copper rich nucleus (see Fig. 12). Their free energies intersect each other in the vicinity of the eutectic composition. In this region, the two types of nuclei are expected to appear simultaneously. We also observed nuclei with alternating $\mathrm{Ag}$ and $\mathrm{Cu}$ rich shells; however, their free energy was considerably higher than that of the "single phase" nuclei displayed here, so they have a negligible probability to appear.

Owing to its complexity, crystal nucleation inside the metastable liquid-liquid miscibility gap will be addressed in the second part of this paper. ${ }^{53}$ We note here only that in the immiscibility region, especially inside the spinodal line, the liquid phase rapidly separates into two liquids of significantly different compositions (coexisting compositions). In this region several types of nuclei compete with each other, including composite nuclei that have a solid core and a liquid "skirt" of a composition between the initial liquid composition and the composition of the crystal.

The results for the Tolman length are shown as a function of temperature in Fig. 13. For the terminal compositions,

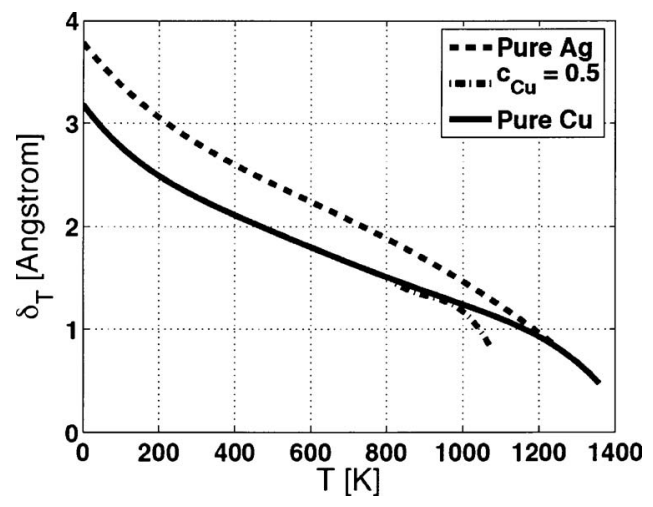

FIG. 13. Tolman length evaluated from the structural order parameter profiles for nuclei shown in Fig. 8. Note the negative temperature coefficient of the Tolman length and its positive limit for planar geometry. 

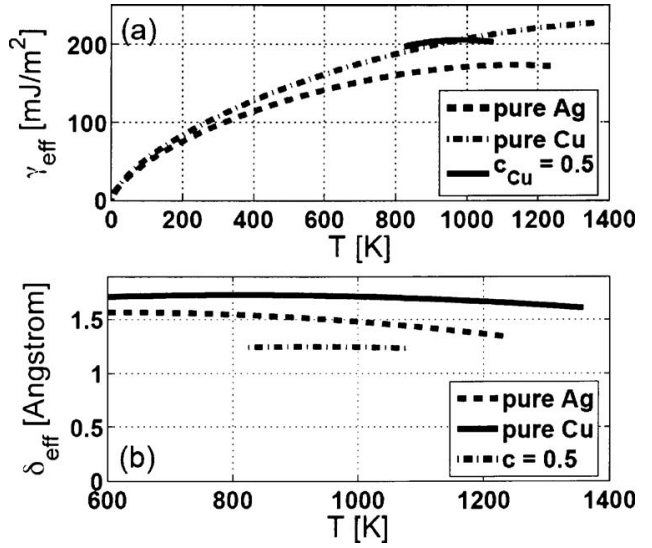

FIG. 14. Effective interfacial free energies and interface thicknesses evaluated from PFT results shown in Fig. 8: (a) effective interfacial free energy and (b) effective interface thickness parameter.

we observe a positive, strongly temperature dependent $\delta_{T}$ that decreases towards a finite positive value corresponding to the equilibrium planar interface with increasing temperature. This behavior is consistent with earlier results obtained with a triple parabolic approximation of the free energy, ${ }^{10(b)}$ which predicts a positive equilibrium value for the Tolman length if the solid-side well of the free energy is steeper than the well on the liquid side, as happens here. The trend of decreasing $\delta_{T}$ with increasing size (temperature) is consistent with results from atomistic simulations. ${ }^{22(b)}$

While the predicted features, such as the asymmetry of the order parameter profiles, the size-dependent interface thickness, and the existence of bulk properties at the center of nuclei, are in a remarkable agreement with more detailed density functional calculations for fcc nucleation, ${ }^{2(b)}$ the assumption of spherical symmetry that we made here excludes the appearance of lamellar or rod-type two-phase structures. Further work is, therefore, needed to clarify whether in the vicinity of the eutectic temperature such nuclei could be more favorable than the single phase nuclei discussed here.

\section{Comparison with other models}

The effective interfacial free energy calculated as $\gamma_{\mathrm{eff}}$ $=\left\{3 W^{*} \Delta g^{2} /(16 \pi)\right\}^{1 / 3}$ and the effective DIT interface thickness $\delta_{\text {eff }}=\left\{3 W^{*} /(4 \pi \Delta g \psi)\right\}^{1 / 3}$ is presented as a function of temperature in Fig. 14. [Inserting $\gamma_{\text {eff }}$ into Eq. (9) of the CNT, and $\delta_{\text {eff }}$ into Eq. (11) of the DIT, one recovers our nonclassical result for the nucleation barrier height.] For the pure components, $\gamma_{\text {eff }}$ is strongly temperature dependent and tends to 0 in the $T \rightarrow 0 \mathrm{~K}$ limit. In contrast to the Tolman length (Fig. 12), the respective $\delta_{\text {eff }}$ is fairly constant in the temperature range of practical importance [see Fig. 14(b)]. Probably, due to the limited temperature range where solution is available (above the metastable liquid coexistence line), at $c=0.5$, the temperature dependence of both $\gamma_{\mathrm{eff}}$ and $\delta_{\text {eff }}$ is less remarkable. These results are in accordance with earlier observation made for small liquid droplets. $^{25(\mathrm{f}), 36(\mathrm{c}), 25(\mathrm{~d})}$ It is also remarkable that bulk crystal properties prevail in the center of nuclei up to fairly large undercoolings $(\sim 500 \mathrm{~K})$, indicating that the main assumptions made in deriving Eq. (11) of the DIT are satisfied.

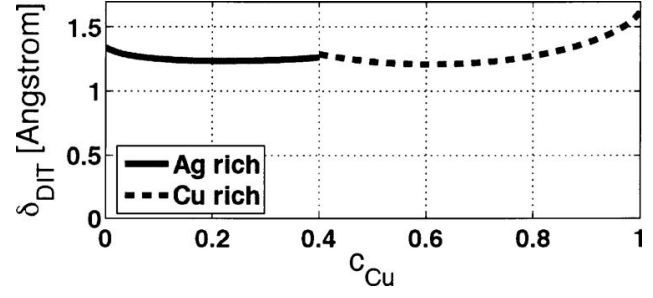

FIG. 15. Composition dependent interface thickness parameter of the DIT.

In order to improve the CNT and DIT predictions, we introduce composition/temperature dependent interfacial parameters for the CNT and DIT that we relate to the free energy of the equilibrium planar interfaces known for the PFT/GL calculations.

(a) We use Eq. (10) to approximate the temperature and composition dependent interfacial free energy in the CNT that postulates that the solid-liquid interfacial free energy is of fully entropic origin.

(b) In the case of DIT, the interface parameter $\delta$ for liquid composition $c$ is evaluated as $\delta(c)=\gamma_{\infty}(c) / \Delta h(T)$, where $\gamma_{\infty}(c)$ is the equilibrium interfacial free energy for a planar interface between a liquid of composition $c$ and a solid of the corresponding solidus composition (shown in Fig. 6), while $\Delta h(T)$ is the respective volumetric enthalpy difference between these liquid and solid phases. The composition dependence of the equilibrium $\delta$ (which is then assumed to be independent of temperature) is shown in Fig. 15.

The temperature dependencies of the interfacial free energy as predicted for pure $\mathrm{Cu}$ by several interface models are compared in Fig. 16. Apparently, making the assumption $\delta$ =const in the DIT approximates better the PFT/GL results than either Eq. (10) or the curvature corrected free energy of the planar interface obtained using the Tolman equation. The PFT calculations performed using the standard double well and interpolation functions (PFT/S) yield nearly linear temperature dependence close to the one Eq. (10) predicts. The difference between the PFT/GL and PFT/S results suggests that it is preferable to use functional forms derived on physi-

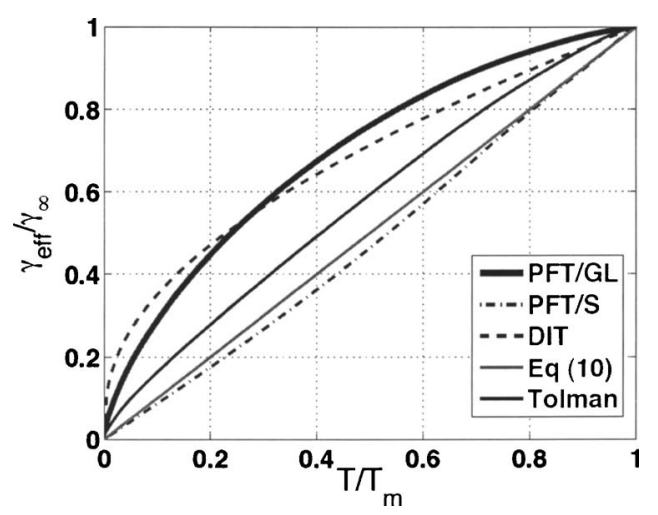

FIG. 16. Temperature dependencies of the interfacial free energy of nuclei as predicted by the phase field theory with Ginzburg-Landau free energy (PFT/GL), by the phase field theory with the standards double-well and interpolation functions (PFT/S), by the phenomenological diffuse interface theory (DIT), by Eq. (10), and by Tolman's expression. 


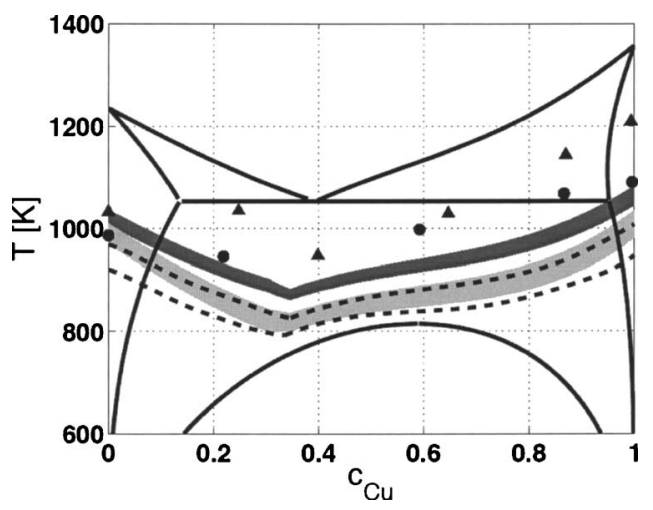

FIG. 17. Range of accessible nucleation rates $\left(10^{-2} \mathrm{~cm}^{-3} \mathrm{~s}^{-1}<J_{\mathrm{ss}}\right.$ $<10^{8} \mathrm{~cm}^{-3} \mathrm{~s}^{-1}$ ) as predicted by several models. [Area bounded by dashed lines, PFT/GL; dark gray band CNT with Eq. (10) and PFT/S; and light gray band, DIT.] Note that the nucleation undercooling predicted by all these models for homogeneous nucleation is considerably larger than that seen in experiment suggesting that in most of the experiments heterogeneous nucleation took place.

cal grounds when available. A recent analysis of nucleation barrier heights based on refined properties of the hard sphere system also prefers PFT/GL to PFT/S. ${ }^{54}$

The accessible range of nucleation rates $\left(10^{-2} \mathrm{~cm}^{-3} \mathrm{~s}^{-1}\right.$ $\left.<J_{\text {ss }}<10^{8} \mathrm{~cm}^{-3} \mathrm{~s}^{-1}\right)$ as predicted by various theories are compared in Fig. 17. In accordance with the results shown in Fig. 16, while the CNT and the PFT/S underestimates the nucleation undercooling for homogeneous nucleation significantly, the DIT prediction is considerably closer to the $\mathrm{PFT} / \mathrm{GL}$ results.

\section{SUMMARY}

The properties of the solid-liquid and solid-solid interfaces and the nucleation of fcc crystalline phase in the eutectic alloy $\mathrm{Ag}-\mathrm{Cu}$ have been investigated using a phase field theory based on a single-order parameter Ginzburg-Landau theory (PFT/GL). We found the following.

(i) The interfacial free energy of the equilibrium planar solid-liquid interface shows a nontrivial behavior both as a function of temperature and composition. In contrast, the interface thickness is roughly independent of temperature.

(ii) Two types of fcc nuclei compete: a $\mathrm{Ag}$ rich and a $\mathrm{Cu}$ rich. For the terminal compositions, the interfacial free energy of critical fluctuations increases with increasing temperature at large undercoolings and levels off at the melting point. At $c=0.5$ the interfacial free energy shows a maximum. The respective interface thickness decreases with temperature for all compositions.

(iii) The Tolman length for the solid-liquid interface is positive for small nuclei and decreases towards a smaller positive value in the planar limit.

(iv) While the DIT predictions for the interfacial free energy of nuclei fall reasonably close to those from the PFT/GL, the CNT with an interfacial free energy from Eq. (10), the PFT/S, and the planar interfacial free energy after Tolman correction seem to underestimate the nucleation undercooling for homogeneous nucleation.

Further work is needed to clarify, however, whether twophase nuclei without spherical symmetry could be competitive with the single phase nuclei investigated here.

\section{ACKNOWLEDGMENTS}

This work has been supported by the Hungarian Academy of Sciences under Contract No. OTKA-K-62588 and by the ESA PECS Contract Nos. 98005, 98021, and 98043.

${ }^{1}$ (a) P. R. ten Wolde, M. J. Ruiz-Montero, and D. Frenkel, Phys. Rev. Lett. 75, 2714 (1995); (b)S. Auer and D. Frenkel, Nature (London) 409, 1020 (2001); (c) B. O’Malley and I. Snook, Phys. Rev. Lett. 90, 085702 (2003); (d) D. Moroni, P. R. ten Wolde, and P. G. Bolhuis, ibid. 94, 235703 (2005).

2 (a) P. Harrowell and D. W. Oxtoby, J. Chem. Phys. 80, 1639 (1984); (b) Y. C. Shen and D. W. Oxtoby, ibid. 105, 6517 (1996); (c) L. Gránásy and T. Pusztai, ibid. 117, 10121 (2002).

${ }^{3}$ For example, see (a) J. Q. Broughton and G. H. Gilmer, J. Chem. Phys. 84, 5749 (1986); (b) B. B. Laird and A. D. J. Haymet, Chem. Rev. (Washington, D.C.) 92, 1819 (1992); (c) J. R. L. Davidchack and B. B. Laird, J. Chem. Phys. 108, 9452 (1998); (d) P. Geysermans, D. Gorse, and V. Pontikis, ibid. 113, 6382 (2000); (e) R. Sibug-Aga and B. B. Laird, Phys. Rev. B 66, 144106 (2002); (f) H. Ramalingam, M. Asta, A. van der Walle, and J. J. Hoyt, Interface Sci. 10, 149 (2002).

${ }^{4}$ J. M. Howe, Philos. Mag. A 74, 761 (1996); P. Schumacher and A. L. Greer, in Light Metals, edited by W. Hale (The Minerals, Metals, and Metaerials Society, Warrendale, PA, 1996), p. 745; W. J. Huisman, J. F. Peters, M. J. Zwanenburg, S. A. de Wries, T. E. Derry, D. Abernathy, and J. F. van der Veen, Nature (London) 390, 379 (1997); J. M. Howe and H. Saka, MRS Bull. 29, 951 (2004); J. F. van der Veen and H. Reichert, ibid. 29, 958 (2004).

${ }^{5}$ For example, see D. W. Oxtoby and A. D. J. Haymet, J. Chem. Phys. 76, 6262 (1982); W. E. McMullen and D. W. Oxtoby, ibid. 88, 1967 (1988); W. A. Curtin, Phys. Rev. Lett. 59, 1228 (1987); , Phys. Rev. B 39, 6775 (1989); N. Choudry and S. K. Gosh, Phys. Rev. E 57, 1939 (1998).

${ }^{6}$ D. W. Oxtoby, Annu. Rev. Mater. Res. 32, 39 (2002).

${ }^{7}$ For recent reviews on the phase field technique see (a) W. J. Boettinger, J. A. Warren, C. Beckermann, and A. Karma, Annu. Rev. Mater. Res. 32, 163 (2002); (b) J. J. Hoyt, M. Asta, and A. Karma, Mater. Sci. Eng., R. 41, 121 (2003); (c) L. Gránásy, T. Pusztai, and J. A. Warren, J. Phys.: Condens. Matter 16, R1205 (2004).

${ }^{8}$ P. R. ten Wolde and D. Frenkel, Science 277, 1975 (1997); , Phys. Chem. Chem. Phys. 1, 2191 (1999).

${ }^{9}$ J. Bechhoefer, H. Löwen, and L. S. Tuckerman, Phys. Rev. Lett. 67, 1266 (1991); L. Gránásy and D. W. Oxtoby, J. Chem. Phys. 112, 2410 (2000).

${ }^{10}$ (a) L. Gránásy, J. Mol. Struct. 485-486, 523 (1999); (b) L. Gránásy and D. W. Oxtoby, J. Chem. Phys. 112, 2399 (2000); (c) L. Gránásy, T. Börzsönyi, and T. Pusztai, Phys. Rev. Lett. 88, 206105 (2002); (d) L. Gránásy, T. Pusztai, T. Börzsönyi, J. A. Warren, B. Kvamme, and P. F. James, Phys. Chem. Glasses 45, 107 (2004).

${ }^{11}$ J. Bragard, A. Karma, Y. H. Lee, and M. Plapp, Interface Sci. 10, 121 (2002); R. Folch and M. Plapp, Phys. Rev. E 68, 010602 (2003).

${ }^{12}$ M. Asta, J. J. Hoyt, and A. Karma, Phys. Rev. B 66, 100101(R) (2002).

${ }^{13}$ L. Gránásy (unpublished).

${ }^{14}$ If the volume integral of a field is constant throughout the time evolution of the system, it is called a conserved field, while the volume integral of nonconserved fields changes with time.

${ }^{15}$ J. A. Warren and W. J. Boettinger, Acta Metall. Mater. 43, 689 (1995).

${ }^{16}$ J. W. Cahn and J. E. Hilliard, J. Chem. Phys. 28, 258 (1958).

${ }^{17}$ S. L. Wang, R. F. Sekerka, A. A. Wheeler, B. T. Murray, S. R. Coriell, R. J. Braun, and G. B. McFadden, Physica D 69, 189 (1993).

${ }^{18}$ B. B. Laird, J. Chem. Phys. 115, 2887 (2001).

${ }^{19}$ R. L. Davidchack and B. B. Laird, J. Chem. Phys. 118, 7651 (2003); B. B. Laird and R. L. Davidchack, J. Phys. Chem. B 109, 17892 (2005).

${ }^{20}$ G. A. Korn and T. M. Korn, Mathematical Handbook for Scientists and Engineers (McGraw-Hill, New York, 1970).

${ }^{21}$ J. W. Cahn and J. E. Hilliard, J. Chem. Phys. 31, 688 (1959). 
${ }^{22}$ For atomistic simulations see (a) W. C. Swope and H. C. Andersen, Phys Rev. B 41, 7024 (1990); (b) L. A. Báez and P. Clancy, J. Chem. Phys. 102, 8138 (1995); for experiment see (c) U. Gasser, E. R. Weeks, A. Schofield, P. N. Pusey, and D. A. Weitz, Science 292, 258 (2001).

${ }^{23}$ D. W. Oxtoby, Liquids, Freezing and Glass Transition, edited by J. P. Hansen, D. Levesque, and J. Zinn-Justin (Elsevier, Amsterdam 1991), p. 145.

${ }^{24}$ J. S. Rowlinson and B. Widom, Molecular Theory of Capillarity (Clarendon, Oxford, 1982).

${ }^{25}$ (a) M. P. A. Fisher and M. Wortis, Phys. Rev. B 29, 6252 (1984); (b) M. Iwamatsu, J. Phys.: Condens. Matter 6, L173 (1994); (c) I. Hadjiagapiou, ibid. 6, 5303 (1994); (d) V. Talanquer and D. W. Oxtoby, J. Phys. Chem. 99, 2865 (1995); (e) K. Koga, X. C. Zeng, and A. X. Shchekin, J. Chem. Phys. 109, 4063 (1998); L. Gránásy, ibid. 109, 9660 (1998); (g) Y. A Lei, T. Bykov, S. Yoo, and X. C. Zeng, J. Am. Chem. Soc. 127, 15346 (2005); (h) E. M. Blokhuis and J. Kuipers, J. Chem. Phys. 124, 074701 (2006).

${ }^{26}$ R. C. Tolman, J. Chem. Phys. 17, 333 (1949).

${ }^{27}$ Tolman's original definition. A different definition is applied by several authors, $\delta_{T}=\lim R \rightarrow \infty R_{e}-R_{p}$. In our terminology, the latter corresponds to the equilibrium Tolman length $\delta_{T, \mathrm{eq}}$.

${ }^{28}$ Defined by the position of a step function that has the same spatial integral and amplitude as the interfacial density profile.

${ }^{29}$ The surface at which the surface tension acts. It is the surface for which the generalized Laplace equation $\Delta p_{c}=2 \gamma / R+\partial \gamma / \partial R$ reduces to the original one, i.e., $\partial \gamma / \partial R=0$.

${ }^{30}$ L. Gránásy, J. Chem. Phys. 104, 5188 (1996); R. McGraw and A. Laaksonen, Phys. Rev. Lett. 76, 2754 (1996).

${ }^{31}$ K. F. Kelton, Solid State Phys. 45, 75 (1991).

${ }^{32}$ B. B. Laird and R. L. Davidchack, J. Phys. Chem. B 109, 17802 (2005).

${ }^{33}$ F. Spaepen and R. B. Meyer, Scr. Metall. 10, 257 (1976).

${ }^{34}$ L. Gránásy, J. Non-Cryst. Solids 162, 301 (1993); Mater. Sci. Eng., A 178, 121 (1994)

${ }^{35}$ L. Gránásy, Europhys. Lett. 24, 121 (1993); J. Phys. Chem. 100, 10768 (1996).

${ }^{36}$ (a) L. Gránásy and F. Iglói, J. Chem. Phys. 107, 3634 (1997); L.
Gránásy, J. Non-Cryst. Solids 219, 49 (1997); (c) L. Gránásy and P. F. James, Proc. R. Soc. London, Ser. A 454, 1745 (1998).

${ }^{37}$ R. S. Aga, J. R. Morris, J. J. Hoyt, and M. Mendelev, Phys. Rev. Lett. 96, 245701 (2006).

${ }^{38}$ K. F. Kelton and A. L. Greer, Phys. Rev. B 38, 10089 (1988); A. L. Greer and K. F. Kelton, J. Am. Ceram. Soc. 75, 1015 (1991).

${ }^{39}$ P. R. ten Wolde, M. J. Kuiz-Montero, and D. Frenkel, J. Chem. Phys. 104, 9932 (1996).

${ }^{40}$ Z. Chvoj, S. Srikanth, and P. Ramachandrarao, J. Non-Equilib, J. NonEquilib. Thermodyn. 24, 360 (1999). The thermodynamic assessment has been done by P. R. Subramanian and J. H. Perepezko [J. Phase Equilib. 14, 62 (1993)].

${ }^{41}$ Y. Waseda and C. H. Miller, Mater. Trans., JIM 19, 546 (1978).

${ }^{42}$ (a) D. Turnbull, J. Chem. Phys. 18, 768 (1950); V. P. Skripov, in Crystal Growth and Materials, Current Topics in Materials Science Vol. 2, edited by E. Kaldish and H. J. Scheel (North Holland, Amsterdam, 1977), p. 327; (c) R. Willnecker, D. M. Herlach, and B. Feuerbacher, Mater. Sci. Eng. 98, 85 (1988).

${ }^{43}$ N. Eustathopoulos, L. Coudurier, J. C. Joud, and P. Desré, J. Cryst. Growth 33, 105 (1976).

${ }^{44}$ D. Y. Sun, M. Asta, and J. J. Hoyt, Phys. Rev. B 69, 174103 (2004).

${ }^{45}$ L. Battezzati and A. L. Greer, Acta Metall. 37, 1791 (1989).

${ }^{46}$ Conclusion drawn from the cross-interfacial density profiles for the (111), (110), and (100) interfaces in the Lennard-Jones system recorded at $0.617,1.0$, and 1.5 reduced temperatures [R. L. Davidchack (private communication)]. Also trivially applies for the hard sphere system.

${ }^{47}$ Y. C. Shen and D. W. Oxtoby, J. Chem. Phys. 104, 4233 (1996); 105, 2130 (1996)

${ }^{48}$ See, for example, L. Gránásy and L. Ratke, Scr. Metall. Mater. 28, 1329 (1993).

${ }^{49}$ G. F. L. Powell, J. Aust. Inst. Met. 10, 223 (1965).

${ }^{50}$ G. F. L. Powell, Trans. Metall. Soc. AIME 245, 1785 (1969).

${ }^{51}$ R. T. Southin and G. A. Chadwick, Acta Metall. 26, 223 (1978).

${ }^{52}$ S. Walder and P. L. Ryder, J. Appl. Phys. 74, 6100 (1993).

${ }^{53}$ G. I. Tóth and L. Gránásy, J. Chem. Phys. 127, 074710 (2007).

${ }^{54}$ G. I. Tóth and L. Gránásy (unpublished). 